\title{
EL PERIFITON COMO INDICADOR DE CONTAMINACIÓN DIFUSA: EL CASO DE LA CUENCA sUPERIOR DEL RÍo Reconquista (Buenos Aires, Argentina)
}

\author{
CAROLINA VILCHES ${ }^{1,2, *}$, MARÍA ADELA CASCO ${ }^{2,3}$ y ADONIS GIORGI ${ }^{1,2}$
}

\begin{abstract}
Summary: Periphyton as indicator of non-point pollution: the case of upper basin of the Reconquista river (Buenos Aires, Argentina). Periphyton in the upper basin of the Reconquista River was studied in order to identify the species that compose and analyze possible changes in its structure due to diffuse pollution from agricultural activities. Six samplings were carried out between December 2006 and May 2008 at intervals of three months in eight reaches adjacent to areas where agricultural and cattle farming activities are developed. Significant differences between the two types of activity were found applying similarity analysis between communities being higher the concentration of chlorophyll-a and lower the autotrophy indexes in agricultural areas. A total of 158 species per reach were found with highly variable species richness (at least 10 species and 67 species maximum). Neither an outstanding dominance by any of the species nor among diversity estimated by Shannon index were found at all sites. The conclusion was that the presence of Ulnaria ulna and Gomphonema parvulum was associated with agricultural sites whereas Nitzschia palea, Raphidiopsis mediterranea and Trachelomonas sp3 were associated with cattle farming.
\end{abstract}

Key words: Periphyton; non-point pollution; Reconquista River; agriculture; cattle farming.

\begin{abstract}
Resumen: Se estudió el perifiton de la cuenca superior del río Reconquista con el objetivo de identificar las especies que lo componen y analizar las posibles modificaciones en su estructura debidas a la contaminación difusa provocada por las actividades agropecuarias. Se realizaron entre diciembre de 2006 y mayo de 2008 seis muestreos a intervalos de tres meses en ocho tramos adyacentes a campos donde se desarrollan actividades agrícolas y ganaderas. Aplicando análisis de similitud entre comunidades se encontraron diferencias significativas entre ambos tipos de actividad siendo más alta la concentración de clorofila-a y menores los índices de autotrofia en zonas agrícolas. Se contabilizaron un total de 158 especies con una riqueza específica muy variable (mínimo 10 especies y máximo 67 especies) por tramo. No se encontró una marcada dominancia por parte de alguna de las especies ni en la diversidad estimada por el índice de Shannon en todos los sitios. Se concluye en cuanto a la composición específica que la presencia de Ulnaria ulna y Gomphonema parvulum se asocia a sitios agrícolas mientras que Nitzschia palea, Raphidiopsis mediterranea y Trachelomonas sp3 a sitios ganaderos.
\end{abstract}

Palabras clave: Perifiton; contaminación difusa; río Reconquista; agricultura; ganadería.

\section{INTRODUCCIÓN}

La agricultura se ha expandido en la Región pampeana argentina en los últimos 50 años a expensas de los paisajes naturales y de las grandes extensiones de pasturas con escaso laboreo. El

1 Departamento de Ciencias Básicas (PEP-INEDES), Universidad Nacional de Luján

2 CONICET

${ }^{3}$ Facultad de Ciencias Naturales y Museo, Universidad Nacional de La Plata

*E-mail: carolina_vilches@hotmail.com incremento en el uso de fertilizantes y pesticidas como consecuencia de esta expansión agrícola, hacen necesario el estudio de su impacto, particularmente porque las condiciones económicas globales conjuntamente con el reciente desarrollo tecnológico agroindustrial han promovido un notorio aumento de la superficie cultivada con soja en la Argentina (Viglizzo et al., 2011). Estas nuevas tecnologías aumentan la rentabilidad de los suelos (Lanson et al., 2009) con el consecuente desplazamiento del ganado vacuno hacia tierras cada vez menos productivas (ej. zona de ribera de 
los arroyos). Esto lleva a una intensificación en el uso de la tierra donde un gran número de animales convive en un área reducida, habitualmente cerca de los cuerpos de agua, o en pasturas implantadas con fertilizaciones periódicas (Hooda et al., 2000).

Las actividades agropecuarias generan diversos tipos de contaminantes cuyo impacto es de difícil evaluación debido a que constituyen una fuente de contaminación difusa, es decir, que ingresa por varios sitios al cuerpo de agua. Este tipo de contaminación puede afectar a las características de los suelos y de las aguas subterráneas y superficiales a la vez que compromete a la biodiversidad presente en los ecosistemas terrestres y acuáticos (Meybeck \& Helmer, 1989). Particularmente, puede provocar cambios en la biomasa y composición taxonómica algal promoviendo efectos de cascadas tróficas que tienen impactos irreversibles sobre la estructura y función de las comunidades acuáticas (Chase, 2003).

El perifiton es una comunidad de organismos que se desarrolla en los cuerpos de agua y está asociada a plantas $\mathrm{u}$ otro sustrato sumergido y que responde predictiva y rápidamente a los cambios en las condiciones ambientales en un gran rango de escalas espaciales (desde metros a decenas de kilómetros) (Gaiser, 2009). Está formada mayoritariamente por individuos sésiles, es generalmente de estructura compacta y los individuos que la constituyen presentan ciclos de vida cortos. Además tiene la capacidad de absorber y concentrar sustancias contaminantes que se encuentren disueltas en el agua. La acción de los contaminantes sobre el perifiton se traduce en cambios en sus características estructurales y funcionales (Economou-Amili, 1980; Aizaki, 1985; Blanck, 1985). Es una comunidad rica en especies y cada especie tiene sus rangos de tolerancia ambiental particulares. Por ello, puede utilizarse como un indicador biológico del cual se obtiene información complementaria a la suministrada por variables físicas y químicas (Cairns \& Niederlehner, 1990; Cairns et al., 1993).

La cuenca del Río Reconquista está asociada a la del Río Luján ya que es adyacente a la misma y desemboca en su tramo final. Si bien en esta última se han desarrollado importantes estudios y relevamientos de la ficoflora planctónica (Del Giorgio et al., 1991; Claps, 1991; Solari \& Claps 1996; O'Farrell et al., 2002, entre otros), la cuenca del Reconquista tiene características particulares tanto hidrológicas, geomorfológicas y de uso de la tierra (Rodriguez Capítulo et al., 2010). Pese a ello, en la cuenca del Reconquista sólo se estudió el fitoplacton en el curso principal (Loez, 1995; Loez \& Topalián, 1999; Rovedatti et al., 2000) donde se registró una disminución de la riqueza de especies en respuesta al estrés producido por contaminación industrial y en los últimos años se ha estudiado la flora bentónica de la cabecera de uno de sus principales afluentes, el arroyo La Choza (Gómez et al., 2009; Licursi et al., 2016). Los objetivos de este trabajo fueron relevar la ficoflora presente en el perifiton de los arroyos de la cuenca superior del río Reconquista y analizar si las modificaciones en su estructura sólo se deben a factores estacionales o pueden también atribuirse a la contaminación difusa que resulta como consecuencia de las actividades agropecuarias.

\section{Material y Método}

\section{Área de estudio}

La cuenca del Río Reconquista está situada en la zona Noroeste de la región metropolitana de Buenos Aires ( $34^{\circ} 41^{\prime} \mathrm{S}$; $59^{\circ} 2^{\prime} 4^{\prime} \mathrm{O}$ ), es un sistema fluvial que discurre por una llanura templada cuyo origen se ubica en la confluencia de los arroyos Durazno, La Choza y La Horqueta, atraviesa varios partidos del gran Buenos Aires y se une finalmente al Río Luján a la altura de San Fernando para desembocar en el Río de La Plata. El área corresponde a la Región pampeana donde la geología se vincula esencialmente con los depósitos sedimentarios Cenozoicos conformados mayoritariamente por limos con cantidades subordinadas de arena y arcilla (Sala \& Auge, 1970). Las pendientes son sumamente bajas $(0,02 \%$ o menores $)$, presenta planicies de inundación restringidas, muy angostas y limitadas a las zonas vecinas a los cauces y no se diferencian terrazas fluviales (Fidalgo, 1983).

Para este estudio se seleccionaron tres sitios de muestreo relacionados a predios con actividad agrícola (La Choza II, La Choza III y Durazno II) y cinco sitios asociados a la actividad ganadera (Arias, Nutrias, La Choza I, Durazno I y Durazno chico) (Fig. 1). Los arroyos La Choza y Durazno reciben la influencia tanto de actividades ganaderas 


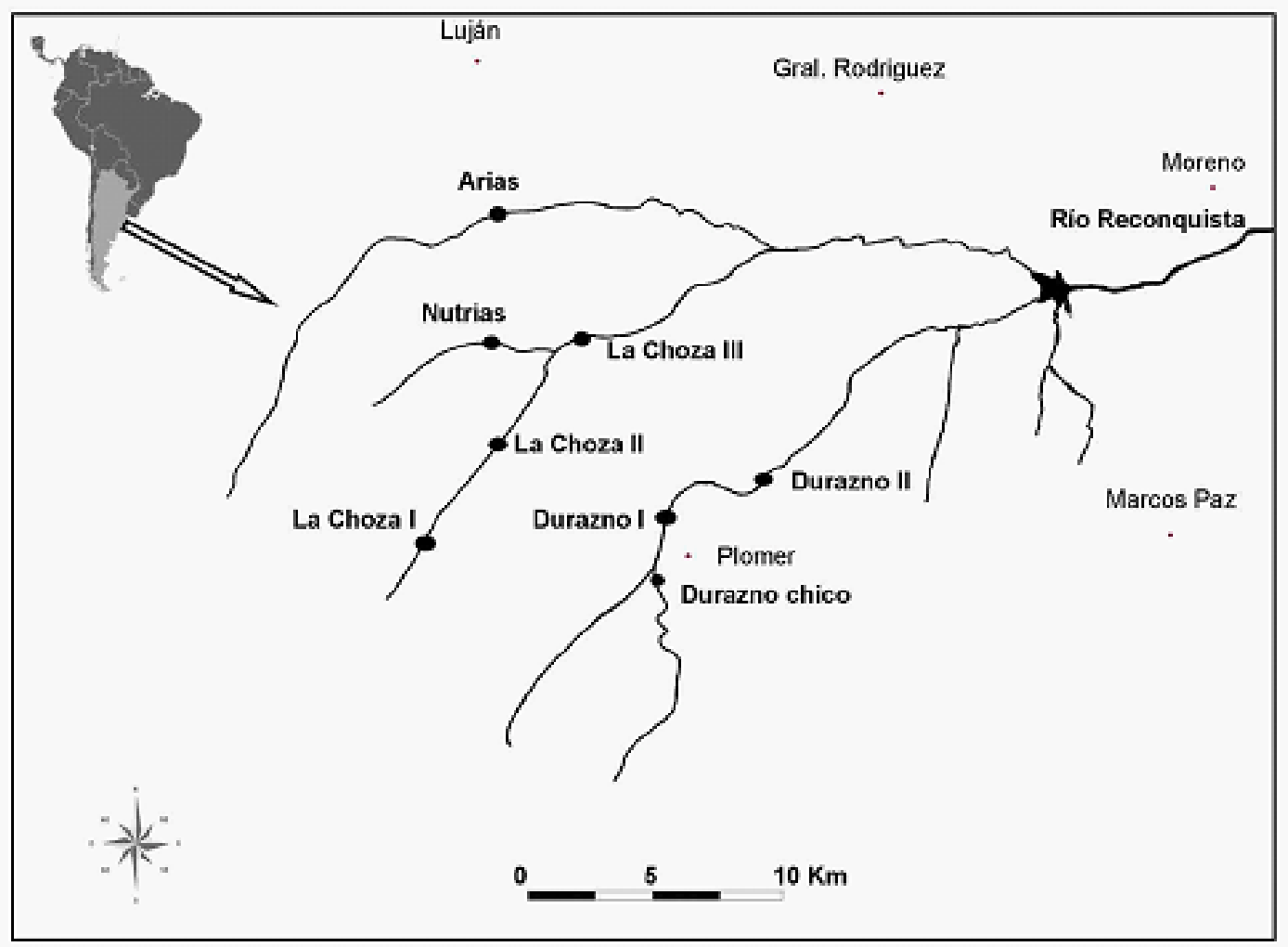

Fig. 1. Mapa con la ubicación de los sitios de muestreo.

como agrícolas mientras que, en las cuencas del arroyo Arias y del arroyo Durazno Chico sólo se desarrolla ganadería. Este último arroyo presenta el menor porcentaje de cobertura de vegetación ribereña y márgenes con mayor pendiente. Se realizaron seis muestreos destinados al estudio del perifiton (diciembre 2006, febrero, mayo, agosto y noviembre 2007 y marzo 2008). Las características hidrológicas y fisicoquímicas del agua fueron publicadas en Vilches et al. (2011) y se resumen en la Tabla 1. Para las muestras de perifiton se seleccionó Ludwidgia peploides (Hunth) Raven, presente en todos los sitios, lo cual permitió minimizar la influencia del sustrato sobre la comunidad analizada. Se obtuvieron trozos de 10 $\mathrm{cm}$ de vástago, evitando extraer los ápices jóvenes y las partes erectas situadas fuera del agua. Cada trozo se colocó en un frasco con agua del acuífero Puelche, previamente colectada libre de cloro. Se tomaron tres réplicas por sitio. Estas muestras fueron conservadas en frío y oscuridad hasta llegar al laboratorio. Allí, en un lapso menor a ocho horas desde la obtención en el campo, las plantas se rasparon con un cepillo de cerdas finas para extraer el perifiton y posteriormente se secaron en estufa a $60{ }^{\circ} \mathrm{C}$ hasta peso constante para estandarizar las estimaciones de perifiton que fueron expresadas en gramo $(\mathrm{g})$ de peso seco de planta.

Las muestras de perifiton así obtenidas fueron homogeneizadas y separadas en alícuotas de $100 \mathrm{ml}$ destinadas para los siguientes análisis: a) biomasa total estimada como peso seco (orgánico e inorgánico) (APHA, 2005), b) concentración de clorofila $a$ y de feopigmentos (Aminot, 1983), c) determinaciones taxonómicas de los grupos algales de acuerdo con la bibliografía específica de cada grupo y siguiendo básicamente los criterios de Cox (1996) para la División Bacillariophyta y Patrick \& Reimer $(1966,1975)$ para la determinación de algunas de sus especies, Komárek \& Anagnostidis (1999, 2005) para las Cyanobacteria, Van Den Hoek et al. (1995) y Graham \& Wilcox (2000) para la División Chlorophyta y Tell \& Conforti (1986) para la División Euglenophyta. 


\begin{tabular}{|c|c|c|c|c|c|c|c|c|c|c|c|c|c|c|c|c|c|c|c|}
\hline 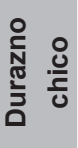 & $\begin{array}{l}8 \\
0 \\
0 \\
+1 \\
0 \\
0 \\
0\end{array}$ & $\begin{array}{l}\stackrel{N}{\check{+}} \\
\frac{+1}{+1} \\
\sigma \\
\sigma\end{array}$ & $\begin{array}{l}\underset{+1}{\mp+1} \\
\stackrel{\infty}{\infty}\end{array}$ & $\begin{array}{l}\llcorner 0 \\
10 \\
0 \\
+1 \\
0 \\
0 \\
0\end{array}$ & $\begin{array}{l}0 \\
0 \\
0 \\
+1 \\
0 \\
0 \\
0 \\
0\end{array}$ & 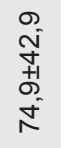 & 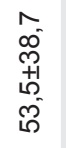 & 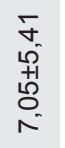 & $\begin{array}{l}\stackrel{N}{\leftarrow} \\
\text { + } \\
\stackrel{+}{1} \\
0 \\
0\end{array}$ & $\begin{array}{l}0 \\
+ \\
+ \\
+ \\
\mathbb{t} \\
\infty\end{array}$ & $\begin{array}{l}\text { N } \\
\text { o } \\
+1 \\
m \\
N \\
N\end{array}$ & $\begin{array}{l}\text { g } \\
+ \\
+1 \\
+\infty \\
\infty \\
\infty \\
1\end{array}$ & 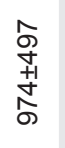 & $\begin{array}{l}0 \\
\text { N } \\
+1 \\
N \\
N\end{array}$ & $\begin{array}{l}8 \\
8 \\
0 \\
+1 \\
o 0 \\
\text { N. } \\
0\end{array}$ & 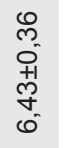 & 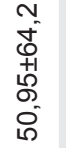 & $\begin{array}{l}m \\
\hat{n} \\
4 \\
\infty \\
0 \\
0\end{array}$ & $\begin{array}{l}\overline{5} \\
\stackrel{0}{0} \\
0 \\
+1 \\
N \\
\infty \\
\infty\end{array}$ \\
\hline 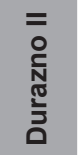 & $\begin{array}{l}\stackrel{N}{0} \\
\stackrel{+}{N} \\
\stackrel{-}{0}\end{array}$ & 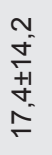 & $\underset{⿱ 亠}{+}$ & $\begin{array}{l}\text { م } \\
0 \\
0 \\
+1 \\
0 \\
-\end{array}$ & $\begin{array}{l}0 \\
\infty \\
+1 \\
0 \\
\stackrel{\infty}{N} \\
\text { N }\end{array}$ & 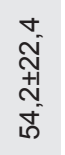 & 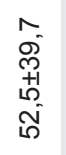 & $\begin{array}{l}\mathbb{N} \\
N \\
N \\
+1 \\
+1 \\
0^{-}\end{array}$ & $\begin{array}{l}\frac{1}{0} \\
0 \\
+1 \\
0 \\
0 \\
0\end{array}$ & $\begin{array}{l}\text { R } \\
\text { N } \\
+1 \\
8 \\
0 \\
0\end{array}$ & $\begin{array}{l}\check{r} \\
\stackrel{+}{+1} \\
\stackrel{+}{N} \\
\stackrel{5}{\leftarrow}\end{array}$ & $\begin{array}{l}8 \\
8 \\
0 \\
+1 \\
+1 \\
N\end{array}$ & $\begin{array}{l}0 \\
\infty \\
m \\
+1 \\
\stackrel{D}{\circ} \\
\sim\end{array}$ & $\begin{array}{l}- \\
\text { Oे } \\
+1 \\
\text { Oे }\end{array}$ & \begin{tabular}{l} 
mo \\
0 \\
0 \\
+1 \\
\multirow{2}{*}{} \\
0 \\
0
\end{tabular} & 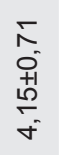 & $\begin{array}{l}\bar{m} \\
\stackrel{+}{+1} \\
\infty 1 \\
\infty \\
\sim\end{array}$ & 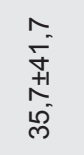 & 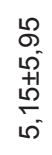 \\
\hline 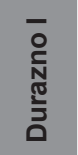 & 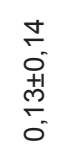 & 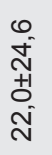 & $\underset{+1}{\stackrel{N}{F}}$ & $\begin{array}{l}\text { 乃 } \\
\stackrel{-}{+} \\
+1 \\
0 \\
0\end{array}$ & 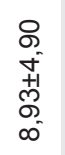 & 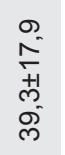 & $\begin{array}{l}0 \\
\tilde{N} \\
\stackrel{+}{+} \\
+0 \\
\infty \\
\infty\end{array}$ & $\begin{array}{l}\infty \\
\stackrel{\infty}{0} \\
\rho^{-1} \\
1 \\
0 \\
0 \\
0 \\
0\end{array}$ & $\begin{array}{l}\stackrel{N}{N} \\
0 \\
+1 \\
0 \\
0 \\
0 \\
0\end{array}$ & 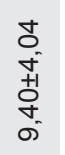 & $\begin{array}{l}\stackrel{2}{S} \\
\text { D } \\
+1 \\
N \\
N\end{array}$ & $\begin{array}{l}\infty \\
\stackrel{0}{0} \\
0 \\
11 \\
\tilde{N} \\
0 \\
N^{-}\end{array}$ & $\begin{array}{l}\stackrel{\mathscr{S}}{2} \\
\stackrel{+}{+1} \\
\stackrel{+}{+}\end{array}$ & $\begin{array}{l}\frac{5}{5} \\
+1 \\
0 \\
0\end{array}$ & $\begin{array}{l}8 \\
0 \\
0 \\
+1 \\
\text { +1 } \\
0 \\
0\end{array}$ & 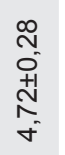 & $\begin{array}{l}0 \\
0 \\
0 \\
+1 \\
0 \\
0 \\
0\end{array}$ & $\begin{array}{l}\infty \\
\infty \\
亡 \\
i n \\
+1 \\
\hat{N} \\
\hat{0}\end{array}$ & $\begin{array}{l}\infty \\
\infty \\
10 \\
1 \\
+1 \\
0 \\
0 \\
\sigma\end{array}$ \\
\hline 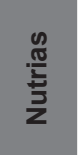 & $\begin{array}{l}\hat{\sigma} \\
\overline{0} \\
+1 \\
\infty \\
\sigma \\
0\end{array}$ & $\begin{array}{l}\infty \\
0 \\
\stackrel{-}{+} \\
\stackrel{+}{+} \\
\stackrel{-}{m}\end{array}$ & $\begin{array}{l}\underset{0}{+1} \\
\stackrel{+1}{\leftarrow}\end{array}$ & $\begin{array}{l}m \\
0 \\
0 \\
+1 \\
10 \\
0 \\
0\end{array}$ & $\begin{array}{l}\bar{m} \\
\stackrel{+}{+1} \\
\stackrel{D}{\sigma} \\
\stackrel{\sigma}{\sigma}\end{array}$ & $\begin{array}{l}\mathfrak{N} \\
\stackrel{N}{N} \\
+1 \\
+ \\
0 \\
0\end{array}$ & $\begin{array}{l}0 \\
\infty \\
+ \\
+1 \\
N \\
N \\
N\end{array}$ & 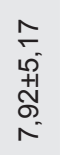 & $\begin{array}{l}m \\
\frac{0}{0} \\
+1 \\
\frac{0}{0} \\
\vdots \\
0\end{array}$ & 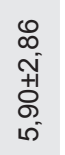 & $\begin{array}{l}0 \\
0 \\
0 \\
0 \\
0 \\
\infty \\
\circ\end{array}$ & $\begin{array}{l}\mathfrak{N} \\
\stackrel{0}{0} \\
+1 \\
N \\
N \\
N\end{array}$ & $\begin{array}{l}\infty \\
0 \\
⿱ \\
+1 \\
+1 \\
\dot{0}\end{array}$ & $\begin{array}{l}\text { N } \\
\text { O } \\
\text { N } \\
\text { O- }\end{array}$ & $\begin{array}{l}\text { 음 } \\
\text { o } \\
+1 \\
\stackrel{+}{N} \\
\text { o. }\end{array}$ & 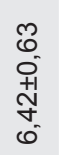 & $\begin{array}{l}\text { 음 } \\
\infty \\
+1 \\
\text { N } \\
\text { in }\end{array}$ & 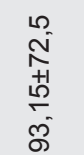 & $\begin{array}{l}m \\
\text { o } \\
\stackrel{+1}{+} \\
\stackrel{m}{5}\end{array}$ \\
\hline$\frac{\mathscr{0}}{\frac{\pi}{2}}$ & $\begin{array}{l}\text { D } \\
\check{0} \\
\text { +1 } \\
\text { N } \\
0 \\
0\end{array}$ & $\begin{array}{l}0 \\
0 \\
\oplus \\
+1 \\
\infty \\
m \\
m\end{array}$ & 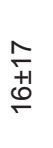 & $\begin{array}{l}\stackrel{d}{N} \\
\stackrel{+}{+} \\
\stackrel{+}{D} \\
\stackrel{-}{-}\end{array}$ & $\begin{array}{l}10 \\
\stackrel{5}{0} \\
+1 \\
D \\
\mathbb{N}\end{array}$ & $\begin{array}{l}\infty \\
\bar{N} \\
+1 \\
0 \\
0 \\
0\end{array}$ & \begin{tabular}{l}
+ \\
\multirow{2}{*}{} \\
+ \\
+1 \\
$\infty$ \\
8 \\
8
\end{tabular} & $\begin{array}{l}\hat{0} \\
+ \\
+1 \\
0 \\
0 \\
\infty\end{array}$ & 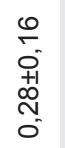 & 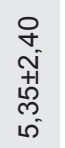 & $\begin{array}{l}- \\
\infty \\
0 \\
+1 \\
+\infty \\
\infty\end{array}$ & $\begin{array}{l}0 \\
0 \\
0 \\
+1 \\
10 \\
10 \\
N\end{array}$ & $\begin{array}{l}0 \\
\dot{+} \\
+1 \\
\stackrel{0}{D} \\
0\end{array}$ & \begin{tabular}{l}
$\bar{N}$ \\
\multirow{1}{*}{} \\
$\infty$ \\
-
\end{tabular} & $\begin{array}{l}\infty \\
\check{-} \\
0 \\
+1 \\
\frac{1}{5} \\
\vdots \\
0\end{array}$ & $\begin{array}{l}+ \\
\infty \\
- \\
+1 \\
\infty \\
\infty \\
0 \\
0\end{array}$ & 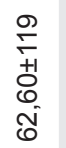 & 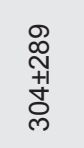 & $\begin{array}{l}\hat{0} \\
\tilde{D}^{-1} \\
0 \\
0 \\
0 \\
0\end{array}$ \\
\hline 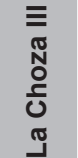 & $\begin{array}{l}F \\
\overline{0} \\
+1 \\
+ \\
\dot{+}\end{array}$ & $\begin{array}{l}0 \\
\bar{N} \\
+1 \\
0 \\
\stackrel{0}{N}\end{array}$ & $\begin{array}{l}\text { N } \\
\text { †े } \\
\text { 亗 }\end{array}$ & $\begin{array}{l}\bar{\delta} \\
+ \\
+1 \\
\stackrel{+}{0} \\
0 \\
-\end{array}$ & $\begin{array}{l}m \\
\tilde{n}^{-} \\
+1 \\
0 \\
N\end{array}$ & $\begin{array}{l}\infty \\
5 \\
5 \\
+1 \\
+ \\
\infty \\
\Gamma\end{array}$ & 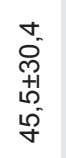 & $\begin{array}{l}\hat{0} \\
+ \\
+1 \\
\stackrel{+}{\sigma} \\
i 0^{-1}\end{array}$ & $\begin{array}{l}\stackrel{N}{\leftarrow} \\
\stackrel{+}{N} \\
\stackrel{+}{\leftarrow} \\
\dot{0}\end{array}$ & 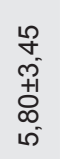 & $\begin{array}{l}0 \\
0 \\
\stackrel{0}{+} \\
\stackrel{+}{1} \\
\stackrel{5}{\Gamma}\end{array}$ & $\begin{array}{l}\text { No } \\
0 \\
0 \\
0 \\
0 \\
0 \\
\end{array}$ & $\begin{array}{l}\infty \\
\infty \\
\infty \\
+1 \\
+ \\
\infty\end{array}$ & $\begin{array}{l}\hat{N} \\
\text { N } \\
m \\
m \\
m\end{array}$ & $\begin{array}{l}\frac{D}{5} \\
0 \\
+1 \\
\infty \\
0 \\
0\end{array}$ & 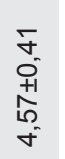 & $\begin{array}{l}8 \\
\stackrel{0}{0} \\
+1 \\
\text { N } \\
\text { N }\end{array}$ & $\begin{array}{l}\stackrel{9}{ } \\
\stackrel{+}{+1} \\
m \\
\stackrel{\sim}{N}\end{array}$ & 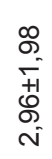 \\
\hline 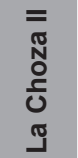 & $\begin{array}{l}10 \\
5 \\
+\infty \\
+1 \\
0 \\
0 \\
0\end{array}$ & \begin{tabular}{l}
10 \\
0 \\
0 \\
+1 \\
0 \\
0 \\
\multirow{1}{1}{}
\end{tabular} & 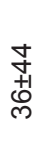 & $\begin{array}{l}\infty \\
\infty \\
0 \\
+1 \\
+ \\
0 \\
-\end{array}$ & 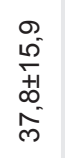 & $\begin{array}{l}0 \\
0 \\
\infty \\
+1 \\
\infty \\
+ \\
+ \\
\infty \\
\infty\end{array}$ & 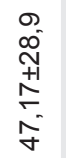 & $\begin{array}{l}m \\
0 \\
0 \\
N \\
N \\
\tilde{N}\end{array}$ & $\begin{array}{l}\circ \\
\circ \\
0 \\
+1 \\
\infty \\
\div \\
0\end{array}$ & $\begin{array}{l}\hat{0} \\
\text { m } \\
\text { 11 } \\
0 \\
6 \\
0\end{array}$ & $\begin{array}{l}0 \\
0 \\
0 \\
m \\
m \\
\stackrel{1}{0}\end{array}$ & $\begin{array}{l}8 \\
0 \\
0 \\
+1 \\
0 \\
0 \\
1\end{array}$ & $\begin{array}{l}8 \\
8 \\
\circ \\
\stackrel{1}{\circ} \\
8 \\
\square\end{array}$ & $\begin{array}{l}- \\
\dot{+} \\
+1 \\
0 \\
\infty\end{array}$ & 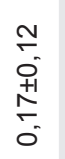 & $\begin{array}{l}\infty \\
\stackrel{+}{0} \\
0 \\
+1 \\
0 \\
+\end{array}$ & 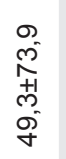 & 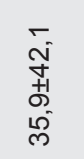 & 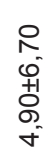 \\
\hline 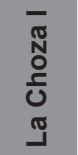 & $\begin{array}{l}E \\
\overline{0} \\
+1 \\
\stackrel{+}{\sigma} \\
0\end{array}$ & 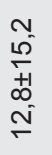 & $\stackrel{\text { O }}{+1}$ & $\begin{array}{l}m \\
m \\
0 \\
+1 \\
N \\
N \\
0\end{array}$ & $\begin{array}{l}\stackrel{N}{\infty} \\
\stackrel{N}{+} \\
\stackrel{+}{+} \\
\stackrel{m}{m}\end{array}$ & $\begin{array}{l}0 \\
\stackrel{0}{0} \\
\stackrel{+}{+1} \\
\stackrel{N}{N} \\
\stackrel{\sigma}{\sigma}\end{array}$ & 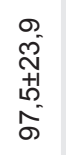 & 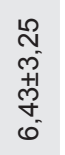 & $\begin{array}{l}\stackrel{N}{\leftarrow} \\
\text { + } \\
+1 \\
\text { N } \\
0\end{array}$ & $\begin{array}{l}+ \\
\tilde{N} \\
+1 \\
\stackrel{+}{0} \\
0 \\
0\end{array}$ & $\begin{array}{l}\infty \\
\infty \\
\infty \\
\infty \\
\infty \\
0 \\
\text { ○े }\end{array}$ & \begin{tabular}{l}
$\hat{N}$ \\
0 \\
+1 \\
\multirow{+}{+}{} \\
$N^{-}$
\end{tabular} & $\underset{\substack{m \\
\stackrel{m}{+}}}{\stackrel{m}{\sigma}}$ & $\begin{array}{l}- \\
\text { ò } \\
\stackrel{1}{N} \\
0\end{array}$ & $\begin{array}{l}F \\
\text { o } \\
+1 \\
\infty \\
\text { m } \\
0\end{array}$ & $\begin{array}{l}\stackrel{N}{-} \\
\text { +1 } \\
\text { N } \\
F\end{array}$ & $\begin{array}{l}\infty \\
\stackrel{0}{0} \\
0 \\
+1 \\
\stackrel{0}{0} \\
\stackrel{1}{0} \\
0 \\
0\end{array}$ & $\begin{array}{l}\hat{\sigma} \\
0 \\
+1 \\
+ \\
\dot{\pi}\end{array}$ & 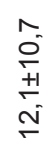 \\
\hline$\frac{\tilde{c}}{\frac{\pi}{\pi}}$ & 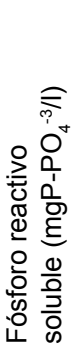 & 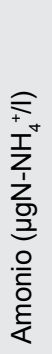 & 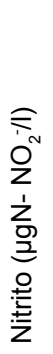 & 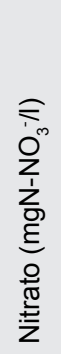 & 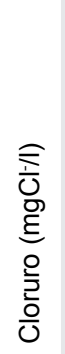 & 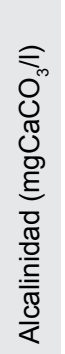 & $\begin{array}{l}\widehat{\sigma} \\
0 \\
\text { O } \\
\xi \\
0 \\
0 \\
0\end{array}$ & 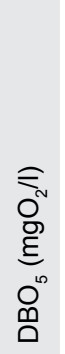 & 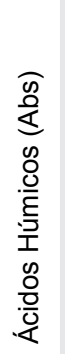 & 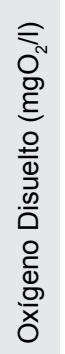 & 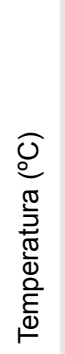 & $\frac{I}{a}$ & 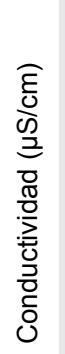 & 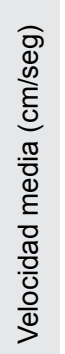 & 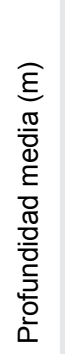 & 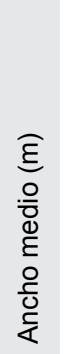 & 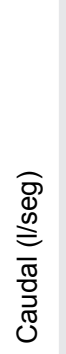 & 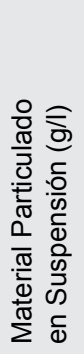 & 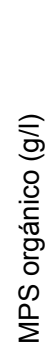 \\
\hline
\end{tabular}




\section{Vilches et al. - El perifiton como indicador de contaminación difusa}

A partir de los parámetros obtenidos se calcularon índices descriptores de la comunidad como el índice autotrófico (IA) (APHA, 2005), el índice de Lakatos (Lakatos, 1989) y la abundancia relativa de cada División de algas y de cianobacterias. Para el cálculo de la riqueza de taxa algales (S), la diversidad de Shannon $\left(\mathrm{H}^{\prime} \log\right.$ en base 2) y la equitatividad de Pielou $\left(\mathrm{J}^{\prime}\right)$ se empleó el paquete DIVERSE del programa Primer $5{ }^{\circledR}$.

Se realizó un análisis de componentes principales con los siguientes descriptores del perifiton: clorofila $a$, feopigmentos, peso seco orgánico, abundancias relativas de Bacillariophyta, Euglenophyta, Chlorophyta y Cyanobacteria y los índices autotrófico, Lakatos clorofila, Lakatos cenizas y $\mathrm{H}^{\prime}$. Las diferencias observadas en cuanto a la composición taxonómica y sus abundancias relativas fueron evaluadas a través del análisis no paramétrico ANOSIM (Primer V5 ${ }^{\circledR}$, Clarke \& Gorley (2001)). Para los análisis estadísticos no se incluyeron los sitios La Choza I y Durazno chico debido a que en éstos no se muestreó el perifiton en todas las fechas (Tabla 2).

\section{Resultados}

En la Tabla 2 se muestran los rangos de variación de los parámetros del perifiton registrados durante todo el período de muestreo. Teniendo en cuenta los resultados obtenidos en todos los arroyos, la concentración de clorofila $a$ fluctuó entre 0,03 y $1,0 \mathrm{mg} / \mathrm{g}$ planta durante los meses del año analizados con excepción del mes de agosto cuando el importante desarrollo de Spirogyra sp. y un incremento en la densidad de diatomeas perifíticas elevó la concentración de clorofila $a$ a valores que llegaron al máximo de $12,75 \mathrm{mg} / \mathrm{g}$ planta en algunos casos. Sin embargo, el arroyo Arias, uno de los sometidos a mayor densidad de ganado en sus márgenes, nunca superó los $0,86 \mathrm{mg} / \mathrm{g}$ planta. Las concentraciones de clorofila $a$ más altas se observaron en agosto sin importar el tipo de arroyo. Comparando los arroyos, se registraron mayores valores relativos de clorofila $a$ en los agrícolas que en los ganaderos.

Durante el período de estudio el IA mostró valores heterotróficos (>200) en un $70 \%$ de los casos para todos los sitios. En agosto los valores de IA disminuyeron a niveles autotróficos salvo en los arroyos Arias y Durazno I. De acuerdo al sistema de Lakatos el perifiton podría clasificarse, en relación al porcentaje de cenizas, como inorgánico en el 97\% de los casos. En relación al porcentaje de clorofila $a$ puede clasificarse como un perifiton de tipo II (auto-heterotrófico) en la mayoría de los sitios. El del arroyo Durazno I sin embargo, se mantuvo como un perifiton hetero-autotrófico y el perifiton del arroyo Arias como netamente heterotrófico. Durante el mes de agosto todos los sitios fueron clasificados como autotróficos (Tabla 2).

La riqueza específica fue muy variable con un mínimo de 10 especies y un máximo de 67 especies en un tramo, hallándose los menores valores durante el mes de agosto. Para el índice de equitatividad de Pielou se encontraron valores cercanos a 1 en la mayoría de los casos lo que implica ausencia de una marcada dominancia por parte de alguna de las especies integrantes del perifiton. La diversidad de Shannon fue alta en todos los sitios (entre 3 y 4 bits/individuo). Los menores valores corresponden al muestreo del mes de agosto donde se registró un marcado desarrollo de Spirogyra. Se encontraron valores cercanos al máximo teórico de diversidad (5 para el índice de Shannon) sin seguir ningún patrón temporal ni relación con los usos de la tierra. En lo que respecta a los grandes grupos taxonómicos algales que conformaron la comunidad perifítica se observó una mayor proporción de Bacillariophyta en los sitios La Choza II, La Choza III, El Durazno I y El Durazno II. Los sitios La Choza I y Arias mostraron mayores valores en Cyanobacteria mientras que el sitio Nutrias estuvo representado por valores semejantes de Bacillariophyta y Euglenophyta y el Durazno chico de Chlorophyta y Cyanobacteria (Tabla 2). El grupo con menor representación en las muestras analizadas fue en general el de las Euglenophyta, con excepción del sitio Nutrias que alcanzó un valor de $32 \%$ durante la primavera. Las Bacillariophyta predominaron durante el otoño y el invierno, mientras que las Euglenophyta tuvieron mayor proporción en los meses cálidos en los sitios ganaderos. Contrariamente, en los sitios agrícolas en verano predominaron las Cyanobacteria (Fig. 2).

La relación entre los distintos parámetros del perifiton se exploró mediante un análisis multivariado de ordenamiento (Fig. 3a). Los dos primeros componentes del Análisis de Componentes Principales (ACP) explican el 32,07\% y $16,67 \%$ 
Bol. Soc. Argent. Bot. 51 (4) 2016

Tabla 2. Rango de variación de los parámetros estructurales del perifiton.

\begin{tabular}{|c|c|c|c|c|c|c|c|c|}
\hline Sitio & La Choza I & La Choza II & La Choza III & Arias & Nutrias & Durazno I & Durazno II & $\begin{array}{l}\text { Durazno } \\
\text { chico }\end{array}$ \\
\hline $\begin{array}{c}\text { Número de } \\
\text { muestras }\end{array}$ & 6 & 18 & 18 & 16 & 18 & 18 & 15 & 6 \\
\hline $\begin{array}{c}\text { Clorofila a } \\
\text { (mg/gplanta) }\end{array}$ & $0,05-1,27$ & $0,08-12,75$ & $0,03-8,78$ & $0,001-0,86$ & $0,08-3,14$ & $0,09-2,09$ & $0,03-8,79$ & $0,12-1,69$ \\
\hline $\begin{array}{l}\text { Feopigmentos } \\
\text { (mg/g planta) }\end{array}$ & $0,0-0,13$ & $0,0-0,07$ & $0,0-0,29$ & $0,0-0,12$ & $0,0-0,30$ & $0,01-0,59$ & $0,0-0,90$ & $0,0-0,04$ \\
\hline $\begin{array}{l}\text { Peso Seco } \\
\text { (g/gplanta) }\end{array}$ & $0,09-0,75$ & $0,06-2,24$ & $0,09-1,54$ & $0,04-0,91$ & $0,03-3,61$ & $0,21-3,2$ & $0,08-4,69$ & $0,35-1,16$ \\
\hline $\begin{array}{c}\text { PS Orgánico } \\
\text { (g/gplanta) }\end{array}$ & $0,03-0,1$ & $0,02-0,46$ & $0,01-0,81$ & $0,008-0,14$ & $0,003-0,55$ & $0,03-0,45$ & $0,01-0,54$ & $0,04-0,14$ \\
\hline $\begin{array}{c}\text { Índice } \\
\text { Autotrófico }\end{array}$ & $80,9-674,1$ & $35,2-478,1$ & $40,9-464,9$ & $161,0-26752$ & $25,0-426,0$ & $107,3-607,3$ & $45,3-362,7$ & $40,7-381,1$ \\
\hline $\begin{array}{c}\text { Lakatos } \\
\text { (\%Cenizas) }\end{array}$ & $65,5-87,8$ & $58,2-83,8$ & $71,5-87,5$ & $67,7-89,3$ & $64,5-98,3$ & $77,1-91,0$ & $67,5-88,4$ & $86,3-88,8$ \\
\hline $\begin{array}{l}\text { Lakatos } \\
(\% \mathrm{Cl}-\mathrm{a})\end{array}$ & $0,15-1,23$ & $0,21-2,84$ & $0,21-2,44$ & $0,003-0,62$ & $0,23-4,0$ & $0,16-0,93$ & $0,27-2,2$ & $0,26-2,45$ \\
\hline Riqueza (S) & $27-67$ & $23-53$ & $10-49$ & $14-38$ & $15-63$ & $20-50$ & $18-63$ & $42-59$ \\
\hline $\begin{array}{l}\text { Equitatividad } \\
\qquad\left(\mathrm{J}^{\prime}\right)\end{array}$ & $0,51-0,83$ & $0,28-0,90$ & $0,51-0,89$ & $0,43-0,83$ & $0,48-0,90$ & $0,39-0,89$ & $0,23-0,91$ & $0,55-0,74$ \\
\hline Diversidad $\left(\mathrm{H}^{\prime}\right)$ & $2,44-4,92$ & $1,29-4,81$ & $1,99-4,76$ & $2,0-4,28$ & $2,35-5,08$ & $1,8-4,77$ & $0,98-5,26$ & $3,13-4,22$ \\
\hline $\begin{array}{c}\text { Bacillariophyta } \\
(\%)\end{array}$ & $8,7-38,86$ & $14,05-97,73$ & $7,0-98,45$ & $0,0-59,27$ & $2,64-87,56$ & $1,22-93,66$ & $29,65-99,49$ & $6,90-34,09$ \\
\hline $\begin{array}{c}\text { Chlorophyta } \\
(\%)\end{array}$ & $1,24-26,83$ & $1,98-45,45$ & $1,54-56,78$ & $5,63-62,93$ & $\begin{array}{l}11,15- \\
54,42\end{array}$ & $3,52-64,08$ & $0,25-24,26$ & $29,0-49,09$ \\
\hline $\begin{array}{c}\text { Cyanobacteria } \\
(\%)\end{array}$ & $12,2-89,75$ & $0,0-64,32$ & $0,0-62,22$ & $11,21-86,71$ & $0,59-43,48$ & $2,46-74,24$ & $0,0-46,54$ & $17,04-49,53$ \\
\hline $\begin{array}{c}\text { Euglenophyta } \\
(\%)\end{array}$ & $0,31-33,11$ & $0,0-16,90$ & $0,0-8,91$ & $0,0-48,15$ & $0,25-78,01$ & $0,0-42,86$ & $0,0-13,48$ & $1,52-19,55$ \\
\hline
\end{tabular}

respectivamente del total de la varianza. El primer componente (autovalor: 3,52) se correlacionó negativamente con la concentración de clorofila $a(-0,92)$, la concentración de feopigmentos $(-0,71)$ y la abundancia relativa de Bacillariophyta $(-0,74)$, y de manera positiva con la abundancia de Cyanobacteria aunque no significativamente $(0,67)$. El segundo componente (autovalor: 1,83$)$ se correlacionó positivamente con la abundancia relativa de Euglenophyta (0,78). Estos resultados indican que la abundancia de Bacillariophyta estaría asociada a altas concentraciones de clorofila $a$ mientras que la abundancia de Cyanobacteria está asociada a condiciones de heterotrofia. La representación de cada uno de los casos en los dos primeros componentes principales se graficaron para visualizar la distribución de cada tipo de actividad (Fig. 3b). No se observa una clara separación entre los sitios ganaderos y agrícolas. Sin embargo, cabe destacar que los puntos ubicados hacia el margen derecho corresponden al sitio ganadero Arias asociado a una mayor abundancia de Cyanobacterias y un mayor índice autotrófico. Por otro lado, los puntos ganaderos ubicados en el tercer cuadrante corresponden al sitio Nutrias. Aunque corresponden al mismo tipo de impacto responden de manera diferente.

La lista de las especies de algas halladas se detalla en la Tabla 3. Se identificaron un total de 159 especies incluyendo los ocho sitios de muestreo. De las especies registradas, 75 pertenecen a la división Bacillariophyta (47,2\%), 46 a la División Chlorophyta $(28,9 \%), 24$ son Cyanobacteria $(15,1 \%)$ y 14 a la división Euglenophyta (8,8\%). El sitio con mayor riqueza de especies fue La Choza II con 116 especies y los de menor riqueza La Choza I y Arias, ambos con 91 especies. De todas las especies halladas la mayoría se encontró indistintamente en los sitios expuestos a la actividad agrícola o ganadera. Las 


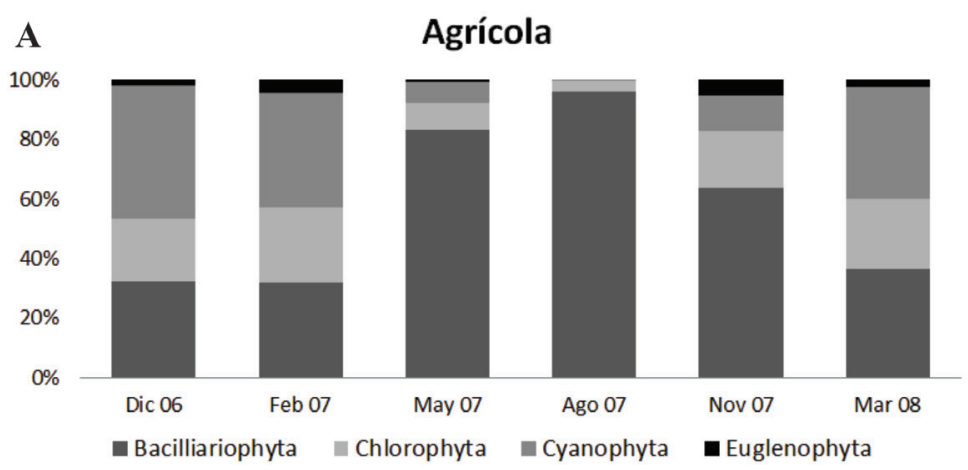

B

Ganadero

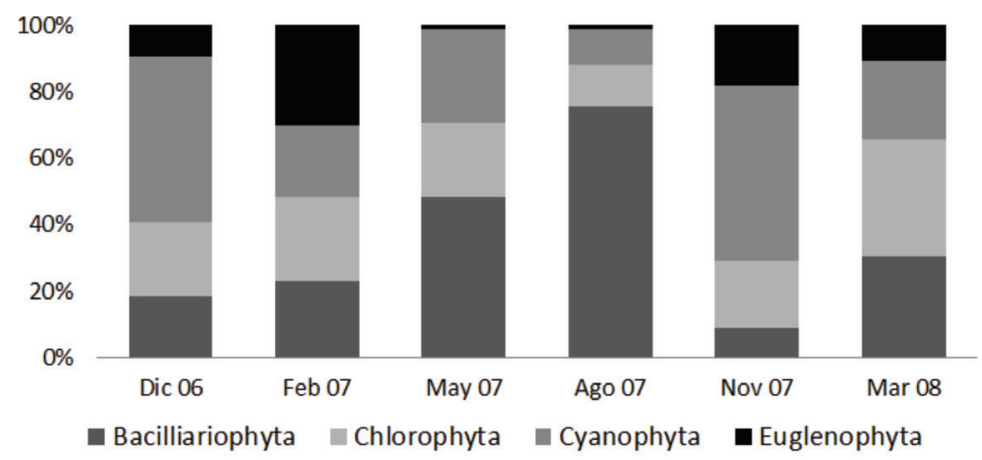

Fig. 2. Composición porcentual de grupos de algas durante el año para los sitios agrícolas (a) y para los sitios ganaderos (b). Período 2006-2008.

especies Gomphonena truncatum, Navicula veneta y Pinnularia sp3 fueron exclusivas de sitios agrícolas mientras que Scenedesmus armatus var. bicauda, Gomphosphaeria sp. y Microcystis aeruginosa fueron exclusivas de sitios ganaderos. Algunas especies se encontraron en sólo uno de los sitios (Tabla 3) como por ejemplo Achnanthes inflata en La Choza II, Korschikoviella gracilipes en Durazno I y Chrooccocus minutus en Durazno II pero éstas se hallaron en una muy baja abundancia relativa, a excepción de Raphidiopsis mediterranea, que se encontró exclusivamente en el arroyo Arias representando la mayoría de las veces un $50 \%$ de la abundancia relativa.

Las diferencias observadas en cuanto a la composición taxonómica y sus abundancias relativas fueron evaluadas a través de un análisis de similitud no paramétrico (ANOSIM) el cual diferenció los dos usos agropecuarios predominantes. El coeficiente $R$ global fue significativo (R: 0,172$)$ con un nivel de significación de $0,1 \%$. Esto permite utilizar las abundancias relativas de las especies encontradas para discriminar aquellas que fueran características de uno u otro tipo de uso mediante el análisis no paramétrico SIMPER basado en los porcentajes de la similitud promedio calculados por el coeficiente de Bray-Curtis y el aporte de cada especie a la segregación o no de un grupo en particular. El valor promedio de similitud obtenido con este análisis de clasificación para el grupo agrícola fue de un 23,6 \%. Para el grupo ganadero, resultó un $23,9 \%$, mientras que la disimilitud media entre ambos tipos de actividad fue de un 79,9\%. Las especies que explican el primer $50 \%$ de disimilitud entre ambos grupos son Lyngbya sp1, Ulnaria ulna, Melosira varians, Oedogonium sp., Rhaphidiopsis mediterranea, Stigeoclonium tenue, Planktothrix sp., Navicula veneta, Gomphonema parvulum, Eunotia pectinalis y Lyngbya sp2. En la Tabla 4 se muestran las especies con mayor representatividad numérica, superior al 
Bol. Soc. Argent. Bot. 51 (4) 2016
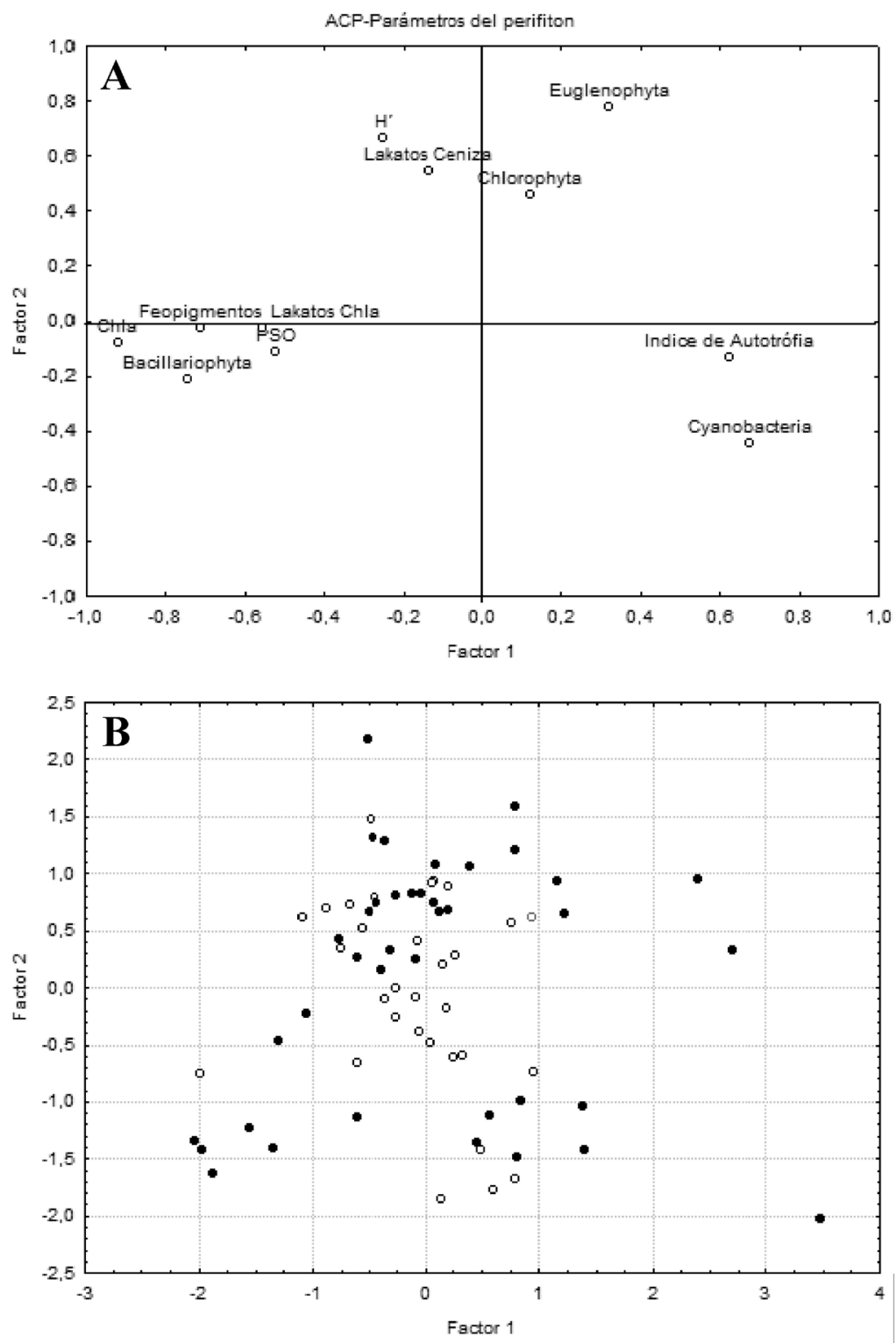

Fig. 3. Ordenamiento en un ACP de (a) las variables del perifiton desarrollado sobre la macrófita Ludwidgia peploides en el período 2006-2008 y (b) cada uno de los casos en el espacio que determinan los dos primeros componentes principales. $(\bullet)$ Sitios ganaderos, $(\circ)$ sitios agrícolas.

$50 \%$, en cada uno de los grupos definidos según el uso de la tierra predominante. En el grupo agrícola, cinco especies fueron mayoritariamente responsables de la similitud dentro del mismo, mientras que en el grupo ganadero aparecen seis especies. La presencia de Ulnaria ulna y Gomphonema parvulum se asoció a sitios agrícolas mientras que Nitzschia palea,
Raphidiopsis mediterranea y Trachelomonas sp3 aparecieron asociadas a sitios ganaderos. Especies como Lyngbya sp1, Oedogonium sp. y Stigeoclonium sp. que aparecen en los dos tipos de agrupamiento, indican poca especificidad por el uso de la tierra adyacente aunque sus abundancias relativas difieren en cada caso. 
Tabla 3. Lista de taxa identificadas. Se señala con una cruz los sitios en que fueron encontradas al menos una vez durante el período de muestreo. ChI = La Choza I, ChII = La Choza II, ChIII = La Choza III, Ar = Arias, Nut = Nutrias, DI = Durazno I, DII = Durazno II, Dch = Durazno chico.

\begin{tabular}{|c|c|c|c|c|c|c|c|c|}
\hline & Chl & Chll & ChIII & $\mathrm{Ar}$ & Nut & DI & D II & Dch \\
\hline \multicolumn{9}{|l|}{ División Bacillariophyta } \\
\hline Achnanthes inflata (Kütz) Grun. & & $\mathrm{X}$ & & & & & & \\
\hline Amphora ovalis Kütz & $\mathrm{X}$ & $\mathrm{X}$ & $\mathrm{X}$ & $\mathrm{X}$ & $\mathrm{X}$ & $\mathrm{X}$ & $\mathrm{X}$ & $x$ \\
\hline Anomoeoneis sphaerophora O. Müll & & $\mathrm{X}$ & $\mathrm{X}$ & $\mathrm{X}$ & $\mathrm{x}$ & & $\mathrm{X}$ & $x$ \\
\hline Aulacoseira granulata var. angustissima (O.Müll.) Simonsen & & $\mathrm{X}$ & & $\mathrm{X}$ & $\mathrm{X}$ & & & \\
\hline Bacillaria paxillifer (O. F. Müller) Hendey & & & $x$ & & & & $\mathrm{x}$ & $x$ \\
\hline Caloneis Cleve sp. & & & & & & & & $\mathrm{x}$ \\
\hline Cocconeis pediculus Ehr. & $x$ & $\mathrm{x}$ & $\mathrm{X}$ & $\mathrm{X}$ & $\mathrm{X}$ & $\mathrm{X}$ & $\mathrm{X}$ & $x$ \\
\hline Cocconeis placentula Ehr. & $\mathrm{X}$ & $\mathrm{X}$ & $\mathrm{X}$ & $\mathrm{X}$ & $\mathrm{X}$ & $\mathrm{X}$ & $\mathrm{X}$ & $x$ \\
\hline Craticula ambigua (Ehr.) D.G. Mann & $\mathrm{X}$ & $\mathrm{X}$ & $\mathrm{X}$ & & & $\mathrm{X}$ & & \\
\hline Cyclostephanos Round sp. & $\mathrm{X}$ & $\mathrm{x}$ & $\mathrm{X}$ & $\mathrm{X}$ & $\mathrm{X}$ & $\mathrm{X}$ & $x$ & $x$ \\
\hline Cylindrotheca closterium (Ehr.) Reiman \& Lewin & & $\mathrm{X}$ & & $\mathrm{X}$ & $\mathrm{x}$ & $\mathrm{X}$ & $x$ & \\
\hline Cymbella minuta Hilse ex Rabh. & $\mathrm{X}$ & $x$ & $\mathrm{X}$ & & $\mathrm{X}$ & $\mathrm{X}$ & $\mathrm{X}$ & \\
\hline Cymbella Agardh sp. & & & & & & $\mathrm{X}$ & & \\
\hline Denticula kuetzingii Grun. & $\mathrm{X}$ & $\mathrm{X}$ & $\mathrm{X}$ & $\mathrm{X}$ & $\mathrm{X}$ & & $\mathrm{X}$ & \\
\hline Diploneis puella (Schum.) Cleve & & & & & $x$ & & $\mathrm{X}$ & \\
\hline Entomoneis Ehr. sp. & & & & & & & & $x$ \\
\hline Epithemia sorex Kütz. & & $\mathrm{X}$ & & & & $x$ & $x$ & \\
\hline Eunotia pectinalis (O.F. Mull) Ralphs. & $\mathrm{X}$ & $\mathrm{X}$ & $\mathrm{X}$ & $\mathrm{X}$ & $\mathrm{X}$ & $\mathrm{X}$ & $x$ & $x$ \\
\hline Eunotia Ehr. sp. & & $\mathrm{X}$ & $\mathrm{X}$ & & $\mathrm{X}$ & $\mathrm{X}$ & $\mathrm{x}$ & \\
\hline Fragilaria capucina Desm. & & & $\mathrm{X}$ & & & $x$ & & \\
\hline Gomphonema affine Kütz. & $\mathrm{X}$ & $\mathrm{x}$ & $\mathrm{X}$ & & $\mathrm{X}$ & $\mathrm{X}$ & $\mathrm{x}$ & $x$ \\
\hline Gomphonema angustatum (Kütz) Rabh. & & $x$ & $\mathrm{X}$ & & $\mathrm{X}$ & $\mathrm{X}$ & & $x$ \\
\hline Gomphonema brasiliense Grun. & $x$ & $x$ & $\mathrm{X}$ & $\mathrm{X}$ & $\mathrm{x}$ & $\mathrm{x}$ & $\mathrm{x}$ & $x$ \\
\hline Gomphonema aff. clavatum Ehr. & $\mathrm{X}$ & $\mathrm{X}$ & $\mathrm{X}$ & & $\mathrm{X}$ & $\mathrm{X}$ & $\mathrm{x}$ & \\
\hline Gomphonema parvulum Kütz. & $\mathrm{X}$ & $\mathrm{X}$ & $\mathrm{X}$ & $\mathrm{X}$ & $x$ & $\mathrm{X}$ & $x$ & $x$ \\
\hline Gomphonema truncatum Ehr. & & $\mathrm{x}$ & & & & & $\mathrm{x}$ & \\
\hline Halamphora veneta (Kütz) Levkov & $x$ & $x$ & $\mathrm{x}$ & & $x$ & $x$ & $\mathrm{X}$ & $x$ \\
\hline Hantzschia amphioxis (Ehr.) Grun. & $x$ & $\mathrm{X}$ & $\mathrm{X}$ & $\mathrm{X}$ & $\mathrm{x}$ & $\mathrm{X}$ & $\mathrm{X}$ & $x$ \\
\hline Hantzschia elongata (Hantzsch) Grunow & & & & & & $x$ & & \\
\hline Hippodonta capitata (Ehr.) Lange-Bert. et al. & & $\mathrm{X}$ & $\mathrm{X}$ & & $\mathrm{X}$ & $\mathrm{X}$ & $\mathrm{x}$ & $x$ \\
\hline Melosira varians C.A. Ag. & $\mathrm{x}$ & $\mathrm{X}$ & $\mathrm{x}$ & $\mathrm{X}$ & $\mathrm{x}$ & $\mathrm{x}$ & & $x$ \\
\hline Navicula Bory sp 1 & & $\mathrm{X}$ & $\mathrm{X}$ & & & & $x$ & $x$ \\
\hline Navicula Bory sp2 & & & $x$ & & $\mathrm{x}$ & $\mathrm{x}$ & $x$ & $x$ \\
\hline
\end{tabular}


Bol. Soc. Argent. Bot. 51 (4) 2016

\begin{tabular}{|c|c|c|c|c|c|c|c|c|}
\hline & $\mathrm{Chl}$ & Chll & ChIII & $\mathrm{Ar}$ & Nut & DI & D॥ & Dch \\
\hline Navicula Bory sp3 & & $x$ & $x$ & $\mathrm{X}$ & & $x$ & $x$ & \\
\hline Navicula Bory sp4 & & & & & & & $x$ & $x$ \\
\hline Navicula Bory sp5 & & $x$ & $x$ & & & & & \\
\hline Navicula Bory sp6 & & $x$ & & & & $\mathrm{X}$ & $x$ & \\
\hline Navicula cryptocephala Kütz. & $x$ & $x$ & $x$ & $x$ & $x$ & $x$ & $x$ & $x$ \\
\hline Navicula peregrina Ehr. & $\mathrm{x}$ & $\mathrm{x}$ & $\mathrm{x}$ & $\mathrm{x}$ & $\mathrm{x}$ & $\mathrm{x}$ & $\mathrm{x}$ & $\mathrm{x}$ \\
\hline Navicula radiosa Kütz. & $x$ & $x$ & $x$ & $\mathrm{X}$ & $x$ & $\mathrm{X}$ & $x$ & $x$ \\
\hline Navicula veneta Kütz. & & $x$ & & & & & $x$ & \\
\hline Neidium iridis (Ehr.) Cleve & $x$ & $x$ & $x$ & $x$ & $x$ & $x$ & $x$ & $x$ \\
\hline Nitzschia Hassal sp1 & $x$ & $x$ & $x$ & & $x$ & $\mathrm{X}$ & $x$ & \\
\hline Nitzschia Hassal sp2 & $x$ & $x$ & $x$ & $x$ & $x$ & $x$ & $x$ & $x$ \\
\hline Nitzschia amphibia Grun. & $\mathrm{x}$ & $\mathrm{x}$ & $\mathrm{x}$ & & $\mathrm{X}$ & $\mathrm{x}$ & $\mathrm{x}$ & $\mathrm{x}$ \\
\hline Nitzschia commutata Grun. & & $\mathrm{x}$ & & $x$ & $\mathrm{X}$ & $x$ & $\mathrm{x}$ & \\
\hline Nitzschia gracilis Hantzsch. & & & $\mathrm{x}$ & $\mathrm{x}$ & & $\mathrm{x}$ & $\mathrm{x}$ & \\
\hline Nitzschia palea (Kütz.) W. Smith & $x$ & $x$ & $x$ & $x$ & $x$ & $x$ & $x$ & $x$ \\
\hline Nitzschia parvula Levis & $\mathrm{X}$ & $\mathrm{x}$ & $\mathrm{x}$ & & $\mathrm{X}$ & $\mathrm{X}$ & $\mathrm{x}$ & \\
\hline Nitzschia recta Hantzsch. & $x$ & $x$ & $x$ & $x$ & $x$ & $x$ & $x$ & $X$ \\
\hline Nitzschia scalpelliformis Grun. & $x$ & $x$ & $x$ & $\mathrm{X}$ & $x$ & $x$ & $x$ & $x$ \\
\hline Nitzschia sigma (Kütz.) W. Smith & & & & & & & $x$ & \\
\hline Nitzschia sigmoidea (Ehr.) W. Smith & $x$ & $x$ & $x$ & $\mathrm{X}$ & $x$ & $x$ & & $x$ \\
\hline Nitzschia subacicularis Hust. & $x$ & $x$ & $x$ & $x$ & $x$ & $x$ & $x$ & $x$ \\
\hline Pinnularia Ehr. sp1 & $x$ & $x$ & $x$ & & $x$ & $x$ & $x$ & $x$ \\
\hline Pinnularia Ehr. sp2 & & $x$ & $x$ & $x$ & $x$ & $x$ & $x$ & $\mathrm{X}$ \\
\hline Pinnularia Ehr. sp3 & & $x$ & & & & & $x$ & \\
\hline Pinnularia braunii Grun. & $x$ & $x$ & $x$ & & $x$ & $x$ & $x$ & $x$ \\
\hline Pinnularia gibba Ehr. & & $x$ & $x$ & $\mathrm{X}$ & $x$ & $\mathrm{x}$ & $x$ & \\
\hline Pinnularia viridis (Nitz.) Ehr. & & $x$ & $x$ & $\mathrm{X}$ & $x$ & $x$ & $x$ & $x$ \\
\hline Placoneis Meresch. sp. & $x$ & $x$ & $x$ & $\mathrm{X}$ & & & & \\
\hline Planothidium Round sp. & $x$ & $x$ & $x$ & & $x$ & $x$ & $x$ & $x$ \\
\hline Pleurosira laevis (Ehr.) Comère & & $\mathrm{x}$ & $x$ & & $x$ & & $x$ & \\
\hline Rhoicosphenia abbreviata (Ag.) Lange-Bertalot & $x$ & $x$ & $x$ & & & $\mathrm{x}$ & $x$ & $x$ \\
\hline Rhopalodia gibba (Ehr.) O. Müller. & & $x$ & & & & & & \\
\hline Rhopalodia gibberula (Ehr.) O. Müller & $\mathrm{x}$ & & $x$ & $\mathrm{X}$ & & & $x$ & $x$ \\
\hline Sellaphora pupula (Kütz.) Mereschk. & & & $\mathrm{x}$ & & $\mathrm{x}$ & & $\mathrm{x}$ & \\
\hline Stauroneis phoenicenteron (Nitz.) Ehr. & $x$ & & & $\mathrm{x}$ & & $x$ & $\mathrm{x}$ & \\
\hline Stephanocyclus meneghiniana (Kütz.) Skabitschevsky & $x$ & $x$ & $x$ & $x$ & $x$ & $x$ & $x$ & $x$ \\
\hline Surirella angustata Kütz. & & & $\mathrm{x}$ & & & $\mathrm{x}$ & $\mathrm{x}$ & \\
\hline
\end{tabular}


C. Vilches et al. - El perifiton como indicador de contaminación difusa

\begin{tabular}{|c|c|c|c|c|c|c|c|c|}
\hline & $\mathrm{Chl}$ & Chll & ChIII & $\operatorname{Ar}$ & Nut & DI & D II & Dch \\
\hline Surirella ovalis Breb. & & $x$ & $x$ & & $x$ & & $x$ & $x$ \\
\hline Surirella robusta Ehr. & & $x$ & $x$ & & $x$ & $x$ & $x$ & $x$ \\
\hline Surirella rorata Frenguelli & & & & & & & & $x$ \\
\hline Surirella tenera Gregory & & $x$ & $x$ & & $x$ & & $x$ & $x$ \\
\hline Ulnaria ulna (Nitz.) Compere & $x$ & $x$ & $x$ & $x$ & $x$ & $x$ & $x$ & \\
\hline \multicolumn{9}{|l|}{ División Chlorophyta } \\
\hline Actinastrum hantzschii Lager. & $\mathrm{X}$ & $x$ & & $x$ & $\mathrm{X}$ & $\mathrm{X}$ & $x$ & $\mathrm{x}$ \\
\hline Ankistrodesmus acicularis (A. Braun) Korshikov & $x$ & $x$ & $x$ & $x$ & $x$ & $\mathrm{x}$ & $x$ & $x$ \\
\hline Ankistrodesmus angustus Bernard & $x$ & $x$ & $x$ & $x$ & $x$ & $x$ & $x$ & $x$ \\
\hline Characium Braun sp. & $x$ & $x$ & $x$ & $x$ & $x$ & $x$ & $x$ & $x$ \\
\hline Chlamydomonas Ehr. sp. & $x$ & $\mathrm{x}$ & $\mathrm{x}$ & $x$ & $x$ & $\mathrm{x}$ & $x$ & $x$ \\
\hline Chodatella Lem. sp. & & & $x$ & $x$ & $x$ & & & \\
\hline Cladophora glomerata (L.) Kütz. & & $x$ & & & & & & \\
\hline Closterium acus (O.Müller) Kütz. & & $x$ & & $x$ & & $\mathrm{x}$ & $x$ & \\
\hline Closterium intermedium Ralfs & $x$ & $x$ & & & $x$ & $\mathrm{x}$ & $x$ & $x$ \\
\hline Closterium parvulum Nägeli & $x$ & & $x$ & & $x$ & & & $x$ \\
\hline Closterium Nitzsch. sp. & $x$ & $x$ & $x$ & $x$ & & & & \\
\hline Coelastrum Nagüeli sp. & $x$ & $x$ & $x$ & $x$ & $x$ & $x$ & $x$ & $x$ \\
\hline Coleochaete Breb. sp. & & $x$ & $\mathrm{x}$ & $x$ & $x$ & $\mathrm{x}$ & $x$ & \\
\hline Cosmarium botrytis Meneghini ex Ralfs & $x$ & $x$ & & & $x$ & $\mathrm{x}$ & & $x$ \\
\hline Cosmarium Corda ex Ralf sp. & $x$ & $x$ & $x$ & $x$ & $x$ & $\mathrm{X}$ & $x$ & $x$ \\
\hline Dicellula Swirenko sp. & $x$ & $x$ & $x$ & $x$ & $x$ & & & $x$ \\
\hline Dictyococcus Gerneck sp. & & & & $x$ & & & $x$ & \\
\hline Euastrum ansatum Ehr. ex Ralfs & & & & & & $x$ & & \\
\hline Euastrum oblongum (Greville) Ralfs ex Ralfs & $x$ & & $x$ & & & & & \\
\hline Eudorina Ehr. sp. & $x$ & $x$ & $x$ & $x$ & $x$ & $x$ & $x$ & $x$ \\
\hline Kirchneriella lunaris (Kirchner) K. Möbius & & $x$ & & & & $\mathrm{x}$ & $x$ & $x$ \\
\hline Korschikoviella gracilipes (Lambert) Silva & & & & & & $x$ & & \\
\hline Micractinium pussillum Fres. & & $\mathrm{x}$ & $\mathrm{x}$ & $x$ & $\mathrm{x}$ & $\mathrm{x}$ & & $\mathrm{x}$ \\
\hline Monoraphidium irregulare (G.M. Smith) Komárková-Legnerová & $\mathrm{x}$ & $\mathrm{x}$ & $\mathrm{x}$ & $x$ & $\mathrm{x}$ & $\mathrm{x}$ & $\mathrm{x}$ & $\mathrm{x}$ \\
\hline Netrium Nägeli sp. & & & & & & & & $x$ \\
\hline Oedogonium Link. sp. & $x$ & $x$ & $x$ & $x$ & $x$ & $x$ & $x$ & $x$ \\
\hline Oocystis Braun sp. & $\mathrm{X}$ & $\mathrm{x}$ & & $x$ & $\mathrm{X}$ & $\mathrm{X}$ & & \\
\hline Pandorina morum (O. Müller) Bory & $x$ & $\mathrm{x}$ & $x$ & & $x$ & $\mathrm{x}$ & $\mathrm{x}$ & $x$ \\
\hline Pediastrum duplex Meyen & $x$ & $x$ & & & & $x$ & & \\
\hline Pediastrum tetras (Ehr.) Ralfs & $x$ & & & & & & & \\
\hline
\end{tabular}


Bol. Soc. Argent. Bot. 51 (4) 2016

\begin{tabular}{|c|c|c|c|c|c|c|c|c|}
\hline & $\mathrm{Chl}$ & Chll & ChIII & $\mathrm{Ar}$ & Nut & DI & D II & Dch \\
\hline Scenedesmus armatus Chodat. & $\mathrm{x}$ & $\mathrm{x}$ & $x$ & $\mathrm{x}$ & $\mathrm{x}$ & $\mathrm{x}$ & $\mathrm{x}$ & $\mathrm{x}$ \\
\hline Scenedesmus armatus var. bicaudatus (Gugl.) Chodat. & & & & $x$ & $x$ & $\mathrm{x}$ & & \\
\hline Scenedesmus falcatus Chodat. & & $\mathrm{x}$ & & $x$ & $\mathrm{x}$ & & $\mathrm{x}$ & $\mathrm{x}$ \\
\hline Scenedesmus flexuosus (Lem.) Ahlstrom & $x$ & $x$ & $x$ & $x$ & $x$ & $\mathrm{x}$ & $x$ & $x$ \\
\hline Schizomeris Kütz sp. & & & $\mathrm{x}$ & & & & & \\
\hline Schroederia setigera (Sch.) Lem. & $x$ & $x$ & $x$ & $x$ & $x$ & $x$ & $x$ & $x$ \\
\hline Spirogyra Link sp. & & $x$ & $x$ & & $x$ & $\mathrm{x}$ & & $x$ \\
\hline Staurastrum Meyen ex Ralph sp. & $x$ & $x$ & $x$ & $x$ & $x$ & $x$ & $x$ & $x$ \\
\hline Staurastrum teliferum Reinsch & & & & & & $x$ & & \\
\hline Stigeoclonium tenue Kütz. & $x$ & $x$ & $x$ & $x$ & $x$ & $x$ & $x$ & $x$ \\
\hline Tetraedron minimum (Braun) Hansg. & $x$ & & & $x$ & $x$ & & $x$ & \\
\hline Tetrastrum Chodat sp. & & $x$ & & $x$ & & $x$ & & \\
\hline Ulotrix Kütz sp. & $x$ & $x$ & $x$ & $x$ & $x$ & $x$ & $x$ & $x$ \\
\hline Xanthidium Ehr. ex Ralf sp. & & & & & & $x$ & & \\
\hline Zygnema Ag. sp. & & & & & $x$ & & & \\
\hline \multicolumn{9}{|l|}{ Division Cyanophyta } \\
\hline Anabaena Bory sp1 & $x$ & $x$ & $x$ & $x$ & $x$ & $x$ & $x$ & $x$ \\
\hline Anabaena Bory sp2 & & & $x$ & & $x$ & & $x$ & $x$ \\
\hline Aphanocapsa Nägeli sp. & $x$ & $x$ & $x$ & & $x$ & $x$ & & \\
\hline Calothrix Ag. sp. & & & & & & $x$ & $x$ & \\
\hline Chroococcus Nägeli sp. & & & & & & $x$ & $x$ & \\
\hline Chrooccocus minutus (Kütz.) Nägeli & & & & & & & $x$ & \\
\hline Gomphosphaeria Kütz. sp. & $x$ & & & & $x$ & & & $x$ \\
\hline Leptolyngbia Anag. sp. & $x$ & $x$ & $x$ & $x$ & $x$ & $x$ & $x$ & $x$ \\
\hline Lyngbia Ag. sp1 & $x$ & $x$ & $x$ & $x$ & $x$ & $x$ & $x$ & $x$ \\
\hline Lyngbia Ag. sp2 & & $x$ & $x$ & $x$ & $x$ & $\mathrm{x}$ & $x$ & $x$ \\
\hline Lyngbia Ag. sp3 & $x$ & $x$ & & & & & & \\
\hline Merismopedia Meyen sp1 & & $x$ & $x$ & $x$ & & $x$ & $x$ & \\
\hline Merismopedia Meyen sp2 & $x$ & $x$ & $x$ & $x$ & $x$ & $x$ & & \\
\hline Microcystis aeruginosa Kütz. ex Lem. & & & & & & $x$ & & $x$ \\
\hline Oscillatoria limnetica Lem. & $x$ & $x$ & $x$ & $x$ & $x$ & $x$ & $x$ & $x$ \\
\hline Oscillatoria princeps Vaucher & $x$ & & $x$ & & $x$ & & & $x$ \\
\hline Oscillatoria tenuis Ag. & $x$ & & $x$ & $x$ & $x$ & $x$ & $x$ & $x$ \\
\hline Oscillatoria Vaucher sp. & $x$ & $x$ & & $x$ & $x$ & $x$ & $x$ & $x$ \\
\hline Phormidium Kütz. ex Gomont sp. & & $x$ & $x$ & $x$ & $x$ & $x$ & $x$ & $x$ \\
\hline Planktothrix Anag. sp. & $\mathrm{x}$ & & $\mathrm{x}$ & $x$ & & $x$ & $\mathrm{x}$ & $\mathrm{x}$ \\
\hline
\end{tabular}




\section{Vilches et al. - El perifiton como indicador de contaminación difusa}

\begin{tabular}{|c|c|c|c|c|c|c|c|c|}
\hline & $\mathrm{Chl}$ & Chll & ChIII & $\operatorname{Ar}$ & Nut & DI & DII & Dch \\
\hline Pseudanabaena limnetica (Lemm.) Komárek & $x$ & $x$ & $x$ & $x$ & $x$ & $\mathrm{x}$ & $x$ & $x$ \\
\hline Raphidiopsis mediterranea Skuja. & & & & $x$ & & & & \\
\hline Spirulina Turpin sp. & $x$ & $x$ & $x$ & $x$ & $x$ & $x$ & $x$ & $x$ \\
\hline Synechococcus Nägeli sp. & & $x$ & $x$ & & $x$ & & & $x$ \\
\hline \multicolumn{9}{|l|}{ División Euglenophyta } \\
\hline Colacium epiphyticum Fritsch. & & & & $x$ & $x$ & & $x$ & \\
\hline Euglena Ehr sp1 & $x$ & $x$ & $\mathrm{x}$ & $\mathrm{x}$ & $x$ & $\mathrm{x}$ & $x$ & $x$ \\
\hline Euglena acus Ehr. & $x$ & $x$ & $x$ & $x$ & $x$ & $x$ & $x$ & $x$ \\
\hline Euglena ehrembergii Klebs & $x$ & $x$ & $x$ & $\mathrm{x}$ & $x$ & $\mathrm{x}$ & $x$ & $x$ \\
\hline Euglena Ehr sp2 & $x$ & $x$ & $x$ & $x$ & $x$ & $x$ & $x$ & $x$ \\
\hline Lepocinclis salina Fritsch & $x$ & $x$ & $x$ & $x$ & $x$ & $\mathrm{x}$ & $x$ & $x$ \\
\hline Lepocinclis caudata Cunha & $x$ & $x$ & $x$ & $x$ & $x$ & $x$ & & $x$ \\
\hline Phacus sp1 & $x$ & $x$ & $x$ & $x$ & $x$ & $x$ & $x$ & $x$ \\
\hline Phacus sp2 & $x$ & $x$ & $x$ & $x$ & $x$ & $x$ & $x$ & $x$ \\
\hline Strombomonas sp. & $x$ & $x$ & $x$ & $x$ & $x$ & $x$ & $x$ & $x$ \\
\hline Trachelomonas sp1 & $x$ & & $x$ & $x$ & $x$ & $\mathrm{x}$ & $x$ & $x$ \\
\hline Trachelomonas sp2 & $x$ & & $x$ & $x$ & $x$ & $\mathrm{x}$ & $x$ & $x$ \\
\hline Trachelomonas sp3 & $x$ & $x$ & $x$ & $x$ & $x$ & $x$ & $x$ & $x$ \\
\hline Trachelomonas sp4 & $\mathrm{x}$ & $\mathrm{x}$ & & $x$ & $x$ & $x$ & $\mathrm{x}$ & $x$ \\
\hline
\end{tabular}

\section{Discusión}

Las actividades agropecuarias generan contaminación difusa, esto significa que pueden incorporar contaminantes al sistema tanto por su línea de costa como en forma aérea a través de fumigaciones o subterránea por drenaje y lixiviación de agroquímicos o nutrientes retenidos temporariamente en suelos, y actúan sobre el conjunto del cuerpo de agua sin que haya una zona definida de ingreso ni un gradiente de deterioro.

En la zona en la que se desarrolló este estudio el epifiton es un componente del sistema que tiene un papel fundamental en el metabolismo de los ríos porque es el mayor responsable de la producción primaria neta superando a las macrófitas (Vilches \& Giorgi, 2010).

La biomasa (estimada como peso orgánico o concentración de clorofila $a$ ) es un indicador estructural utilizado a nivel mundial. Sin embargo, no parecería ser un indicador muy fiable del grado de calidad química del agua ya que este parámetro tiene una fuerte dependencia de las condiciones hidráulicas y de la disponibilidad de luz (Montuelle et al., 2010). En nuestro estudio, posiblemente debido a que entre los tramos analizados dichas características ambientales fueron semejantes, una de las principales características que aparece como diferencia de los arroyos agrícolas respecto de los ganaderos es la concentración de clorofila $a$. Las comunidades de arroyos que pasan cerca de campos agrícolas tienen valores de clorofila $a$ promedio mayores que aquellos donde se realiza ganadería. Resultados de este tipo fueron destacados también por otros autores (Heinonen, 1984; Urrea \& Sabater, 2009). Esta diferencia debido al uso no enmascara la componente estacional, observándose en agosto mayores 
Tabla 4. Especies que contribuyeron a la similitud dentro de cada grupo agropecuario. En negrita se destacan las especies que pertenecen únicamente a uno de los grupos.

\begin{tabular}{|ll|}
\hline \multicolumn{1}{|c|}{ Grupo agrícola } & \multicolumn{1}{c|}{ Grupo ganadero } \\
\hline Lyngbya sp1 & Lyngbya sp1 \\
Ulnaria ulna & Oedogonium $\mathrm{sp}$. \\
Oedogonium sp. & Trachelomonas sp3 \\
Stigeoclonium tenue & Stigeoclonium $\mathrm{sp}$. \\
Gomphonema parvulum & Raphidiopsis mediterranea \\
& Nitzschia palea \\
\hline
\end{tabular}

valores de clorofila $a$ en todas las muestras del perifiton debido en gran parte al marcado desarrollo de Spirogyra. Esta estacionalidad también está reflejada en el Índice de autotrofia (IA) que indica en agosto el paso desde una comunidad predominantemente heterotrófica (debido a las altas concentraciones de materia orgánica en el agua, las que permitirían el mayor desarrollo de heterótrofos y acumulación de detritos) a una autotrófica. Giorgi \& Feijoó (2010) mostraron que los cambios más importantes en el grado de autotrofia del perifiton de un arroyo pampeano estarían asociados a la pluviosidad, aumentando la heterotrofia por incremento de materia orgánica detrítica depositada por arrastre. En nuestro caso, sin embargo, la variación del índice de autotrofia fue estacional ya que se redujo en agosto por el excesivo desarrollo de algas filamentosas que ocurre previamente a las estaciones más lluviosas (Giorgi, 1998). Lo mismo se observa por el sistema de Lakatos que indica un perifiton de tipo inorgánico o inorgánicoorgánico como consecuencia del elevado porcentaje de material inorgánico que se depositaría en la matriz del perifiton proveniente de los sólidos en suspensión típicamente abundantes en este tipo de ambientes (Pizzarro \& Alemanni, 2005).

En nuestra zona de estudio pocos investigadores han trabajado en algas de cuerpos lóticos de la zona (Loez \& Topalián, 1999; Gómez \& Licursi, 2001, Licursi et al., 2016 entre otros) por lo que nuestros resultados contribuyen a incrementar información básica existente sobre la ficoflora de estos ambientes que podrá utilizarse para comparar con resultados de estudios futuros.
Durante el período analizado la riqueza específica promedio hallada en los sitios ganaderos fue menor que en los sitios agrícolas. Esto coincide con observaciones de Middleton (2010) quien encontró que el acceso del ganado al cuerpo de agua afecta significativamente la composición de la comunidad perifítica y que la riqueza en los sitios donde no hay acceso de ganado es $15 \%$ mayor a los sitios con acceso. Este autor también atribuye a una mala calidad del agua la presencia de las diatomeas Navicula cryptocephala y $N$. radiosa, algunas cianobacterias filamentosas del género Oscillatoria y los euglenoideos Trachelomonas. Estos organismos, a pesar de estar presentes en los sitios que estudiamos no se asociaron a ningún uso específico de la tierra, excepto Trachelomonas sp3 que contribuye a aumentar la similitud de los sitios ganaderos. En concordancia con este hallazgo, Garduño Solórzano et al. (2011) manifiestan que el género Trachelomonas es un típico indicador de medianas a altas concentraciones de materia orgánica en el agua y está especialmente asociado con altas concentraciones de amonio.

Tell \& Conforti (1986) también destacan que muchos euglenoideos son particularmente abundantes en aguas estancadas ricas en materia orgánica y de alto contenido trófico como los sitios cercanos a tierras agrícolas o los expuestos a fecas animales. En este estudio, si bien se encontraron euglenoideos en todos los sitios, fueron muy abundantes en el arroyo Nutrias, uno de los sitios con ganado y con velocidad de corriente más baja, con valores de DQO similares a los otros sitios ganaderos $\left(73,2 \mathrm{mgO}_{2} / \mathrm{l}\right)$ y valores de DBO de 7,92 $\mathrm{mgO}_{2} / 1$.

Nitzschia palea es considerada por LangeBertalot y Watanabe (Round, 1993) como heterotrófica y una de las especies más tolerantes a contaminación severa por nutrientes. $N$. palea además es la segunda especie en orden decreciente de tolerancia a la contaminación orgánica según Palmer (1969) y en el índice de diatomeas pampeano se la ubica en la categoría que indica peor calidad y mayor grado de disturbio (Gomez \& Licursi, 2001). Esta especie aparece como importante en la similitud de los sitios ganaderos en este estudio. Por otro lado, Raphidiopsis mediterranea, exclusiva del sitio ganadero Arias (con altos contenidos 
de material particulado en suspensión y ácidos húmicos que reducen la intensidad lumínica), es considerada por Reynolds et al. (2002) como tolerante a condiciones de baja intensidad lumínica. Esta especie es planctónica al igual que Microcystis aeruginosa y Scenedesmus armatus var. bicauda y fueron encontradas en el perifiton de sitios ganaderos únicamente favorecidos indudablemente por las altas concentraciones de nutrientes y la escasa velocidad de la corriente de agua que permiten su desarrollo y supervivencia como especies ocasionales en el epifiton.

En los sitios agrícolas se encontró un perifiton auto-heterotrófico con mayor variación estacional y mayor riqueza específica que en los sitios ganaderos, y donde las algas que más aportan a la similitud son Ulnaria ulna y Gomphonema parvulum, ambas especies señaladas como tolerantes a la contaminación por nutrientes (Round, 1993). Claps (1991) menciona a $G$. parvulum como abundante durante todo el año así como para del Giorgio et al. (1991) es una de las especies más persistentes. Estos autores también mencionan a $U$. ulna como resistente a las crecidas y al incremento en la turbulencia del agua. Licursi et al. (2016) en un experimento de enriquecimiento de nutrientes mencionan a ambas especies como abundantes y frecuentes aunque $U$. ulna es considerada mesotrófica.

Debido a estos antecedentes es importante destacar que los arroyos de la zona presentan altas concentraciones de materia orgánica y sustancias húmicas y niveles de fósforo y nitrógeno que imponen a las aguas características de eutrofia como se observa en la Tabla 1 (Vilches et al., 2011). Es por ello que la presencia de actividades ganaderas o agrícolas pareciera favorecer la mayor o menor abundancia relativa de determinados grupos más que un cambio cualitativo en la composición taxonómica. Probablemente las mayores diferencias entre tramos que atraviesan campos agrícolas o ganaderos se den en aquellos casos donde se permite el ingreso de animales (Arias, Nutrias, Durazno I) que aportan además materia orgánica y realizan otra serie de perturbaciones directamente dentro del cuerpo de agua como pisoteo, resuspensión de sedimentos que interfiere en la cantidad de luz incidente, herbivoría de macrófitas eliminando también parte de las epifitas y reduciendo los sustratos disponibles para la colonización. De este modo se ven afectadas claramente la biomasa y riqueza del perifiton.

Tanto la ganadería como la agricultura serían fuentes de producción de compuestos de fósforo y nitrógeno aunque sólo podrían diferenciarse químicamente estas actividades teniendo en cuenta las fracciones orgánica e inorgánica de estos parámetros. El ganado puede incorporar la fracción orgánica de dichos nutrientes directamente al agua o pueden ingresar posteriormente por escorrentía mientras que los campos agrícolas incorporarían por escorrentía la fracción inorgánica excedente de la fertilización.

\section{Conclusiones}

En base a los muestreos realizados se concluye que existen diferencias entre las zonas agrícolas y ganaderas, dadas principalmente por mayores valores de clorofila $a$ y menores índices autotróficos en los sitios agrícolas. En cuanto a la composición específica, parte de las especies encontradas han sido señaladas como de ambientes con mucha materia orgánica y alta eutrofia por otros autores. Particularmente, la presencia de Ulnaria ulna y Gomphonema parvulum se asoció a sitios agrícolas mientras que Nitzschia palea, Raphidiopsis mediterranea y Trachelomonas sp3 aparecieron asociadas a sitios ganaderos.

\section{Agradecimientos}

A L. Ferrari, P. Castagné, M. Mastrángelo, F. de la Torre, P. Scarcia y N. Ossana por la colaboración en el trabajo de campo. Además a la Dra. V. Conforti por la colaboración en la determinación de euglenoideos y a dos revisores que mejoraron sustancialmente la primera versión de este manuscrito. Este estudio fue apoyado financieramente por la Agencia Nacional de Promoción Científica y Tecnológica de Argentina (ANPCyT), PICT N ${ }^{\circ}$ 26165. C. Vilches tuvo una beca del Consejo de Investigaciones Científicas y Técnicas de Argentina (CONICET) durante la realización del proyecto. 


\section{Biblografía}

AIZAKI, M. 1985. Removal and excretion of dissolved organic matter by periphyton community grown in eutrophic river water. Jap. J. Limnol. 46: 159-168.

APHA-WWA-WPCF. 2005. Standard methods for the examination of water and wastewater. Washington DC.

BLANCK, H. 1985. A simple, community level, ecotoxicological test system using samples of periphyton. Hydrobiologia 124: 251-261.

CAIRNS, J. \& B. R. NIEDERLEHNER. 1990. Biological monitoring of Hazardous Waste sites. Comments toxicol. 3: 493-508.

CAIRNS J., P. V. McCORMICK \& B. R. NIEDERLEHNER. 1993. A proposed framework for developing indicators of ecosystem health. Hydrobiologia 263: 1-44.

CLAPS, M. 1991. Diatom communities on aquatic macrophytes of pampasic lotic environments (Argentina). Acta Hydrobiol. 33: 195-208.

CLARKE, K. \& R. GORLEY. 2001. Primer V5: User manual/tutorial. Primer-E, Plymouth.

CHASE, J. 2003. Strong and weak trophic cascades along a productivity gradient. Oikos 101: 187-195.

DEL GIORGIO, P., A. VINOCUR, R. LOMBARDO \& G. TELL. 1991. Progressive changes in the structure and dynamics of the phytoplankton community along a pollution gradient in a lowland river - a multivariate approach. Hydrobiologia 224: 129-154.

ECONOMOU-AMILLI, A. 1980. Periphyton analysis for the evaluation of water quality in running waters of Greece. Hydrobiologia 74: 39-48.

FIDALGO, F. 1983. Algunas características de los sedimentos superficiales en la cuenca del Río Salado y en la Pampa Ondulada. Coloquio internacional sobre hidrobiología de grandes llanuras, pp. 10451066.

GAISER, E. 2009. Periphyton as an indicator of restoration in the Florida Everglades. Ecol. Indicators 9: 37-45.

GARDUÑO SOLÓRZANO, G., M. G. OLIVA MARTÍNEZ, A. LUGO VÁZQUEZ, M. B. MENDOZA GARFIAS, R. QUINTANAR ZUÑIGA \& V. CONFORTI. 2011. Trachelomonas (Euglenophyta) from a eutrophic reservoir in Central Mexico. J. Environm. Biol. 32: 46-471.

GIORGI, A. 1998. Factores reguladores del fitobentos en arroyos de llanura. Tesis doctoral, Universidad Nacional de La Plata.

GIORGI, A. \& C. FEIJOÓ. 2010. Variación temporal de la biomasa del perifiton de Egeria densa Planch en un arroyo pampeano. Limnetica 29: 269-278.

GÓMEZ N. \& M. LICURSI. 2001. The Pampean Diatom Index (IDP) for assessment of rivers and streams in Argentina. Aquatic Ecol. 35: 173-181.
GÓMEZ, N., M. V. SIERRA, J. COCHERO, M. LICURSI \& D. BAUER. 2009. Epipelic biofilms as indicators of environmental changes in lowland fluvial systems. In: BALEY, W. (Ed.), Biofilms: formation, development and properties, pp. 259-290. Nova Science Publisher.

HEINONEN, P. 1984. Early warning of eutrophication in rivers by analysis of periphyton chlorophyll a. In: PASCOE, D. \& R. EDWARDS (Eds.), Freshwater biological monitoring, pp. 45-52. Pergamon Press, New York.

HOODA, P., A. EDWARDS, H. ANDERSON \& A. MILLER. 2000. A review of water quality concerns in livestock farming areas. Sci. Total Environm. 250: 143-167.

LAKATOS, G. 1989. Composition of reed periphyton (biotecton) in the Hungarian part of lake Fertö. Biol. Forschung für Burg. 71: 125-134.

LANSON, D., L. SCHEIN \& M. MIGLIORANZA. 2009. Aportes para la comprensión de la incidencia de los factores climáticos y tecnológicos sobre la deriva de agroquímicos aplicados a cultivos de soja y sus respectivos efectos sobre la población potencialmente expuesta. In: PONVERT, D. \& D. BATISTA (Eds.), Seminario Internacional "La tecnología satelital de observación de la tierra en la evaluación, monitoreo y manejo de desastres naturales en la agricultura. Retos y perspectivas", pp. 69-79. Ediciones INTA, Buenos Aires.

LICURSI, M., N. GOMEZ \& S. SABATER. 2016. Effects of nutrient enrichment on epipelic diatom assemblages in a nutrient-rich lowland stream, Pampa Region, Argentina. Hydrobiologia 766: 135150.

LOEZ, C. 1995. Estudios limnológicos en el río Reconquista (Provincia de Buenos Aires): relación entre parámetros biológicos y químicos, especialmente el impacto del zinc sobre la estructura del fitoplancton. Tesis doctoral, Universidad de Buenos Aires.

LOEZ, C. \& M. TOPALIÁN. 1999. Use of algae for monitoring rivers in Argentina with a special emphasis for the Reconquista river (región of Buenos Aires). In: PRYGIEL, J., B. WHITTON \& J. BUKOWSKA (eds), Use of algae for monitoring rivers III, pp. 72-83. Agence de 1'Eau ArtoisPicardie, Douai.

MEYBECK, M. \& R. HELMER. 1989. The quality of rivers: From pristine stage to global pollution. Global Planet. Change 1: 283-309.

MIDDLETON, R. 2010. Cattle access affects periphyton community structure in Tennessee farm ponds. Master's Thesis, University of Tennessee.

MONTUELLE, B., U. DORIGO, A. BÉRARD, B. VOLAT, A. BOUCHEZ, A. TLILI, V. GOUY \& 


\section{Vilches et al. - El perifiton como indicador de contaminación difusa}

S. PESCE. 2010. The periphyton as a multimetric bioindicator for assessing the impact of land use on rivers: an overview of the Ardières-Morcille experimental watershed (France). Hydrobiologia 657:123-141.

O'FARRELL, I., R. LOMBARDO, P. DE TEZANOS PINTO \& C. LOEZ. 2002. The assessment of water quality in the Lower Luján River (Buenos Aires, Argentina): phytoplankton and algal bioassays. Environm. Pollut. 120: 207-218.

PALMER, C. 1969. A composite rating of algae tolerating organic pollution. J. Phycol. 5: 78-82.

PIZARRO, H. \& M. E. ALEMANNI. 2005. Variables físico-químicas del agua y su influencia en la biomasa del perifiton en un tramo inferior del Río Luján (Provincia de Buenos Aires). Ecol. Austral 15: 73-78.

REYNOLDS, C., V. HUSZAR, C. KRUK, L. NASELLIFLORES \& S. MELO. 2002. Towards a functional classification of the freshwater phytoplankton. $J$. Plank. Res. 24: 417-428.

RODRIGUES CAPITULO, A., N. GOMEZ, A. GIORGI \& C. FEIJOÓ. 2010. Global changes in pampean lowland streams (Argentina): implications for biodiversity and functioning. Hydrobiologia 657 : 53-70.

ROUND, F. 1993. A review and methods for the use of epilithic diatoms for detecting and monitoring changes in river water quality (Methods for the examination of waters and associated materials). Her Majesty's Stationery Office, London.

ROVEDATTI, M., C. LOEZ, M. TOPALIÁN, P. CASTAÑE, A. SALIBIAN \& H. OLGUíN. 2000. Monitoring of a polluted river (Reconquista, Argentina) based on physicochemical parameters and phytoplankton. Verh. Internat. Verein Limnol. 27: 2743-2748.
SALA, J. \& M. AUGE. 1970. Algunas características geohidrológicas del noreste de la provincia de Buenos Aires. Cuartas Jornadas Geológicas Argentinas, Tomo III.

SOLARI, L. \& M. CLAPS. 1996. Planktonic and benthic algae of a pampean river (Argentina): comparative analysis. Ann. Limnol. Int. J. Lim. 32: 89-95.

TELL, G. \& V. CONFORTI. 1986. Euglenophyta pigmentadas de la Argentina. J. Cramer, Berlin.

URREA, G. \& S. SABATER. 2009. Epilithic diatom assemblages and their relationship to environmental characteristic in an agricultural watershed (Guadiana River, SW Spain). Ecol. Indicators 9: 693-703.

VIGLIZZO, E., F. FRANK, L. CARREÑO, E. JOBBAGY, H. PEREYRA, J. CLATT, D. PINCÉN \& M. F. RICARD. 2011. Ecological and environmental footprint of 50 years of agricultural expansion in Argentina. Global Change Biol. 17: 959-973.

VILCHES, C. \& A. GIORGI. 2010. Metabolism in macrophyte-rich stream exposed to flooding. Hydrobiologia 654: 57-65.

VILCHES, C., A. GIORGI, M. MASTRÁNGELO \& L. FERRARI. 2011. Non-point contamination homogenizes the water quality of Pampean streams. Bull. Environm. Contam. Toxicol. 87: 147-151.

Recibido el 26 de octubre de 2015, aceptado el 18 de marzo de 2016. 
